\title{
The Association Between Sagittal Plane Alignment and Disc Space Narrowing of Lumbar Spine in Farmers
}

\author{
Ki Hoon Park, $\mathrm{MD}^{1}$, Sora Baek, MD, PhD ${ }^{1,2}$, Eun Kyoung Kang, MD, PhD ${ }^{1,2}$, \\ Hee-won Park, $\mathrm{MD}^{1,2}$, Gowun Kim, MD, $\mathrm{PhD}^{1,2}$, Sung Hyun Kim, MD, $\mathrm{PhD}^{3}$
}

\begin{abstract}
${ }^{1}$ Department of Rehabilitation Medicine, Kangwon National University School of Medicine, Chuncheon; ${ }^{2}$ Center for Farmers' Safety and Health and Department of Rehabilitation Medicine, Kangwon National University Hospital, Chuncheon; ${ }^{3}$ Human Medical Imaging and Intervention Center, Seoul, Korea
\end{abstract}

Objective To investigate whether lumbar lordosis (LL) and lumbar segmental lordosis (LSL) are related to sex, age, low back pain (LBP), and lumbar disc space narrowing (DSN).

Methods A total of 569 farmers were recruited. In lateral spine radiograph, LL (L1-L5) and LSL (L1, L2, L3, L4, and L5) were measured using Cobb's method. The differences in LSL values ( $\triangle \mathrm{LSL}$ ) according to the presence or absence of a DSN were calculated as $\mathrm{LSL}_{\mathrm{DSN}}-\mathrm{LSL}_{\mathrm{noDSN}}$ for each DSN level.

Results In male, the lateral spine radiograph showed significantly greater L4-LSL and L5-LSL and smaller L1-LSL and L2-LSL compared to female. LLs in the 50-59 and $\geq 60$ years age groups were significantly smaller compared to those in the $<50$ years age group. In subjects with LBP, LL and L4-LSL were significantly smaller than in those without. The $\Delta$ LSLs at the disc level with DSN showed the greatest decrease: L1- $\Delta$ LSL $\left(\Delta-3.99^{\circ}\right)$, L2- $\Delta$ LSL $(\Delta$ $\left.-3.31^{\circ}\right)$, L3- $\Delta \operatorname{LSL}\left(\Delta-2.87^{\circ}\right)$, L4- $\Delta \operatorname{LSL}\left(\Delta-3.31^{\circ}\right)$, and L5- $\Delta$ LSL $\left(\Delta-4.44^{\circ}\right)$ in L1/2, L2/3, L3/4, L4/5, and L5/S1 DSN, respectively. Conversely, distant $\Delta$ LSLs were inversely increased: L1-LSL $\left(\Delta 0.75^{\circ}\right)$ with $\mathrm{L} 4 / 5$ DSN and L2-LSL $\left(\Delta 0.94^{\circ}\right)$ with L5/S1 DSN.

Conclusion Sagittal plane alignment was significantly associated with sex, age, LBP, and DSN. LSLs around the levels of DSN were decreased, and there was compensational increase of LSL distant to the DSN to maintain the overall LL.

Keywords Low back pain, Intervertebral disc degeneration, Lordosis, Lumbar vertebrae

Received February 18, 2021; Revised June 16, 2021; Accepted July 1, 2021; Published online August 30, 2021

Corresponding author: Sora Baek

Department of Rehabilitation Medicine, Kangwon National University Hospital, Kangwon National University School of Medicine, Baengnyeong-ro 156, Chuncheon 24289, Korea. Tel: +82-33-258-9100, Fax: +82-33-258-2146, E-mail: sora.baek@kangwon.ac.kr

ORCID: Ki Hoon Park (https://orcid.org/0000-0003-3516-3136); Sora Baek (https://orcid.org/0000-0003-3404-6202); Eun Kyoung Kang (https://orcid. org/0000-0001-5315-1361); Hee-won Park (https://orcid.org/0000-0002-7434-6675); Gowun Kim (https://orcid.org/0000-0002-5694-8111); Sung Hyun Kim (https://orcid.org/0000-0002-4142-3021).

(c) This is an open-access article distributed under the terms of the Creative Commons Attribution Non-Commercial License (http://creativecommons.org/ licenses/by-nc/4.0) which permits unrestricted noncommercial use, distribution, and reproduction in any medium, provided the original work is properly cited. Copyright $\odot 2021$ by Korean Academy of Rehabilitation Medicine 


\section{INTRODUCTION}

Lumbar lordosis (LL) is a natural inward curvature of the lumbar spine in the sagittal plane. The sacral slope (SS), the angle between the horizontal line and the cranial sacral endplate, is an important parameter associated with spinopelvic balance. LL is a remarkable aspect of the human body that facilitates upright posture and bipedal walking [1]. When the human body evolved for upright bipedal walking, the human spine was repositioned to a new location and LL developed to facilitate efficient bipedal walking [1]. The lumbar sagittal alignment can be useful in evaluating the balance between gravity and muscular activity [2].

It has been reported that the loss of LL is a critical sign of back problems. The association between decreased LL and low back pain (LBP) and lumbar spinal disorders has gained the attention of many researchers [3-6]. Restoration of LL is one of the goals of lumbar fusion surgery to achieve favorable outcomes [7].

A decrease in LL has been reported in many lumbar disc disorders including lumbar disc degeneration [6], lumbar disc herniation $[8,9]$, and degenerative lumbar spinal stenosis [10]. In a prospective study with the development of degenerative scoliosis, reduction of lumbar segmental lordosis (LSL) more than $5^{\circ}$ was identified at the same level of segmental scoliotic wedge [11]. These intervertebral disc disorders are related with disc space narrowing (DSN) on lumbar radiograph. DSN appeared more strongly associated with back pain than other radiographic features [12]. However, the association between the segment of lumbar disc disorders and adjacent segmental lordosis changes have not yet been investigated.

In order to assess the association between sagittal plane alignment and lumbar spinal disorders, it is necessary to know the factors affecting sagittal plane alignment. LL is known to be associated with sex and age, although there are controversial results. Some studies argued that male and older individuals had smaller LL, while other studies said that there no relationship existed between LL with sex or age [5,13-18]. In this study, we aimed to investigate the differences in lumbar sagittal alignment according to sex and age.

Though a supine lateral lumbar spine radiograph is considered an acceptable means of measuring lordotic angles [19], the optimal position for the radiologic measurement of lordosis is standing [20]. Most LL studies use radiographs taken in the standing position, reporting this as the most functionally relevant position [20]. The most common evaluation of LL measures the angle formed by all five lumbar segments (L1-L5): the upper line is drawn at the cranial endplate of $\mathrm{Ll}$ and the lower line at the cranial endplate of the sacrum (S1) [20]. Measurement of LL and LSL using the Cobb method includes human error in drawing lines. The Cobb method has become the gold standard in measuring LL [20] and is shown to have high interobserver and intraobserver reliability [21]. However, a limitation of measuring LL using the Cobb method is that the two spinal curvatures of different segmental magnitudes may result in the same LL angle [20]. In an attempt to overcome this problem, we decided to measure lordosis by lumbar segment.

Inconsistent results have been reported regarding the relationship between LL and sex, age, LBP, and DSN. The relationship between the change in LSL and disc height changes is little known. We aimed to determine whether LL, LSL, and SS are related to sex, age, LBP, and lumbar DSN.

\section{MATERIALS AND METHODS}

\section{Subjects}

Participants were recruited in two ways: 446 eligible subjects were selected from the Farmers' Cohort for Agricultural Work-Related Musculoskeletal Disorders study conducted from 2013 to 2014 and 339 new participants were selected from individuals farming in Gangwon Province. After excluding non-active farmers, 590 farmers participated. Twenty-one subjects were excluded from the analysis due to history of spine surgery. Ultimately, 569 farmers, aged 39-82 years, were included in the final analysis. Farming types were classified into rice farming, greenhouse farming, dry field farming, and orchard farming.

This study was approved by the Institutional Review Board of Kangwon National University Hospital (No. 2016-03-008) and written informed consent was obtained from all of the participants. This study was also registered with the Clinical Research Information Service. 


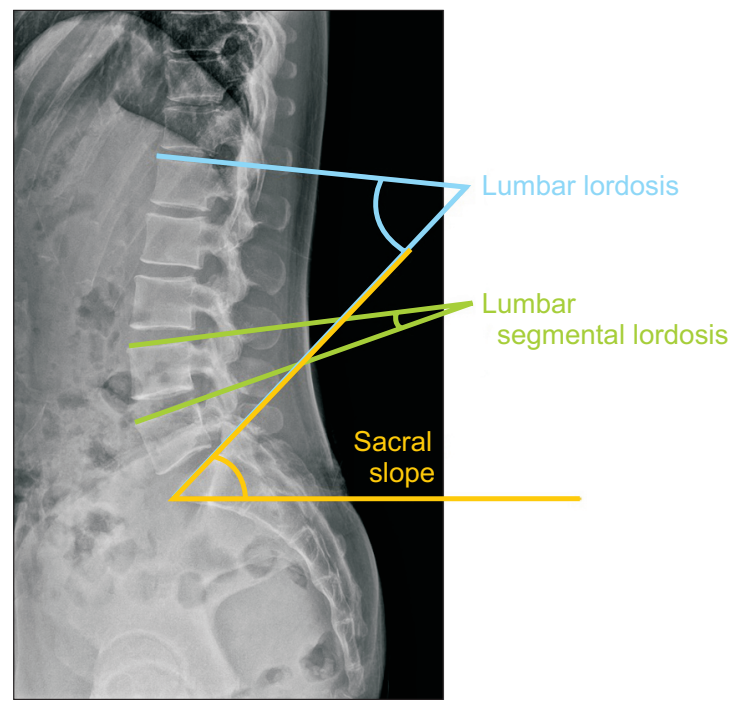

Fig. 1. Measurement techniques for assessment of lumbar lordosis, lumbar segmental lordosis, and sacral slope.

\section{Sagittal alignment of lumbar spine}

Spine radiographs were obtained with subjects in a neutral standing position in the lateral view. Subjects were assessed by parameters such as LL, LSL, and SS. We defined LL as the angle between the cranial end plate of L1 and the cranial end plate of S1, using Cobb method on lateral lumbar X-ray; LSL of each lumbar segment was evaluated as the angle between the cranial endplates of adjacent vertebrae using Cobb method described as L1 (angle between the cranial end plates of L1 and L2), L2 (angle between the cranial end plates of L2 and L3), L3 (angle between the cranial end plates of L3 and L4), L4 (angle between the cranial end plates of L4 and L5), and L5 (angle between the cranial end plates of L5 and S1), respectively; and SS as the angle between the horizontal line and the cranial sacral end plate tangent [22,23] (Fig. 1).

Second measurements were performed with more than a 2-week interval by the same observer to investigate the reliability of LL, LSLs, and SS. Intraobserver reliability was calculated by interclass correlation coefficients (ICCs). To determine the degree of agreement for the paired measurements, Dunn's classification of ICCs were used (moderate, 0.41-0.6; substantial, 0.61-0.8; almost perfect, $>0.81)$ [24].

\section{Lumbar DSN}

Assessment of disc space of L1/2, L2/3, L3/4, L4/5, and
L5/S1 in lateral spine radiograph was done by a specialized expert radiologist who was blinded to the subject's information. Disc height was assessed as percent (\%) of adjacent discs and graded as semi-quantitative scoregrade 0 , normal; grade 1 , mild $(>75 \%)$; grade 2 , moderate $(>50 \%)$; grade 3 , severe $(>25 \%)$; grade 4 , very severe $(<25 \%)$ - as described by Mimura et al. [25]. Disc height with grade 1 or more was considered as the presence of DSN. The differences in LSL values ( $\Delta$ LSL) according to the presence or absence of a DSN were calculated as $\mathrm{LSL}_{\mathrm{DSN}}-\mathrm{LSL}_{\mathrm{noDSN}}$ for each DSN level from $\mathrm{L1} / 2$ to $\mathrm{L} 5 / \mathrm{S} 1$.

\section{LBP}

LBP was assessed by a questionnaire (KOSHA CODE H-30-2003) [26], which was modified by the Korea Occupational Safety and Health Agency based on the National Institute for Occupational Safety and Health (NIOSH) questionnaire, the survey questionnaire used by the NIOSH (United States) [27]. The questionnaire inquired about the presence of LBP in the past year, duration $(<1$ day, $<1$ week, $<1$ month, $<6$ months, or $\geq 6$ months) and its severity (mild, moderate, severe, or extreme) and frequency (once in 6 months, once in 2 or 3 months, once a month, once a week, or daily). We defined clinically meaningful LBP as LBP that lasted more than 1 week or occurred more frequently than once a month with a severe degree of pain in the past 1 year.

\section{Statistical analysis}

To compare the LL, LSL, and SS between male and female, we performed a Student t-test. A one-way analysis of variance (ANOVA) was performed to compare three age groups: $<50$ years, $50-59$ years, and 60 years or more. Post hoc comparisons were made with Dunnett's test for comparisons between age groups. The comparison of LL, LSLs, and SS values according to the presence of LBP and L1/2 to L5/S1 DSN were analyzed using a Student t-test. Correlation between lumbar sagittal alignment and grade of L1/2 to L5/S1 DSN were calculated using Spearman's correlation test. A p-value of $<0.05$ was considered statistically significant. All statistics were analyzed using SPSS version 21.0 (IBM, Armonk, NY, USA). 


\section{RESULTS}

\section{Subject characteristics}

Of all subjects, 259 (45.5\%) were male and $310(54.5 \%)$ were female. The average age of subjects was $58.11 \pm 7.07$ years. All subjects were actively working farmers. The proportion of farming types were rice farming $(7.9 \%)$, greenhouse farming $(43.1 \%)$, dry field farming $(38.7 \%)$, and orchard farming $(10.4 \%)$. The average farming period was 26.8 years.

\section{Lumbar sagittal alignment}

The ICC values for the paired measurements were between 0.882 and 0.971 (LL, 0.971; L1-LSL, 0.882; L2-LSL, 0.911; L3-LSL, 0.930; L4-LSL, 0.937; L5-LSL, 0.931; SS, $0.944)$, indicating an almost perfect intra-observer reliability according to Dunn's classification.

The average LL was $49.8^{\circ}$. L5-LSL accounted for the largest proportion of LL, which was $20.4^{\circ}$ ( $41 \%$ of LL), followed by L4-LSL, which was $12.6^{\circ}$ (25\% of LL). L4 and L5 segments accounted for a total of $66 \%$ of LL. The average SS was $34^{\circ}$ (Table 1 ).

\section{Sex and lumbar sagittal alignment}

There were no differences in LL and SS between male and female. However, there were statistically significant differences in LSLs between male and female, except for L3-LSL. In male, the segmental lordotic angle was significantly greater in L4 and L5 segments, whereas it was significantly smaller in L1 and L2 segments (Table 1).

\section{Age and lumbar sagittal alignment}

There were significant differences in LL between age groups, and LSLs from L2, L3, and L4 segments. The SS also showed a tendency to decrease according to age; however, this was not statistically significant (Table 2).

Table 1. Sex and lumbar sagittal alignment

\begin{tabular}{|cccccc}
\hline & Proportion (\%) & All subjects $(\mathbf{n = 5 6 9 )}$ & Male $(\mathbf{n = 2 5 9 )}$ & Female (n=310) & p-value \\
\hline LL & & $49.8 \pm 11.81$ & $50.4 \pm 10.6$ & $49.3 \pm 12.8$ & 0.239 \\
\hline L1-LSL & 4 & $1.9 \pm 4.32$ & $1.4 \pm 4.2$ & $2.4 \pm 4.4$ & $0.004^{* *}$ \\
\hline L2-LSL & 12 & $5.8 \pm 3.98$ & $5.3 \pm 3.6$ & $6.3 \pm 4.2$ & $0.003^{* *}$ \\
L3-LSL & 18 & $8.8 \pm 3.96$ & $8.5 \pm 3.5$ & $9.0 \pm 4.3$ & 0.182 \\
L4-LSL & 25 & $12.6 \pm 4.85$ & $13.1 \pm 4.2$ & $12.2 \pm 5.3$ & $0.024^{*}$ \\
L5-LSL & 41 & $20.4 \pm 6.48$ & $22.0 \pm 5.9$ & $19.1 \pm 6.6$ & $<0.001^{* *}$ \\
SS & & $34.0 \pm 7.95$ & $33.8 \pm 7.2$ & $34.2 \pm 8.6$ & 0.465 \\
\hline
\end{tabular}

Values are presented as mean \pm standard deviation.

LL, lumbar lordosis; LSL, lumbar segmental lordosis; SS, sacral slope.

${ }^{*} \mathrm{p}<0.05,{ }^{* *} \mathrm{p}<0.01$.

Table 2. Age and lumbar sagittal alignment

\begin{tabular}{|c|c|c|c|c|c|c|c|}
\hline & \multicolumn{3}{|c|}{ Age group } & \multicolumn{4}{|c|}{ p-value } \\
\hline & \multirow{2}{*}{$<50(n=75)$} & \multirow{2}{*}{$50-59(n=224)$} & \multirow{2}{*}{$\geq 60(n=270)$} & \multirow{2}{*}{ ANOVA } & \multicolumn{3}{|c|}{ Dunnett post hoc test } \\
\hline & & & & & $<50$ vs. 50-59 & $<50$ vs. $\geq 60$ & $50-59$ vs. $\geq 60$ \\
\hline LL & $53.8 \pm 9.5$ & $50 \pm 10.7$ & $48.5 \pm 13.0$ & $0.001^{* *}$ & $0.014^{*}$ & $<0.001^{* *}$ & 0.376 \\
\hline L1-LSL & $2.4 \pm 3.5$ & $2.3 \pm 4.1$ & $1.5 \pm 4.7$ & 0.080 & - & - & - \\
\hline L2-LSL & $7.2 \pm 3.2$ & $5.9 \pm 3.8$ & $5.4 \pm 4.2$ & $<0.001^{* *}$ & $0.013^{*}$ & $<0.001^{* *}$ & 0.393 \\
\hline L3-LSL & $9.7 \pm 3.3$ & $8.9 \pm 3.5$ & $8.4 \pm 4.4$ & $0.014^{*}$ & 0.203 & $0.012^{*}$ & 0.312 \\
\hline L4-LSL & $14.3 \pm 3.7$ & $12.3 \pm 4.9$ & $12.3 \pm 5.0$ & $<0.001^{* *}$ & $0.001^{* *}$ & $0.001^{* *}$ & 0.998 \\
\hline L5-LSL & $19.8 \pm 6.4$ & $20.4 \pm 6.2$ & $20.6 \pm 6.7$ & 0.622 & - & - & - \\
\hline SS & $35.7 \pm 7.4$ & $33.6 \pm 7.6$ & $33.9 \pm 8.4$ & 0.130 & - & - & - \\
\hline
\end{tabular}

Values are presented as mean \pm standard deviation.

LL, lumbar lordosis; LSL, lumbar segmental lordosis; SS, sacral slope.

${ }^{*} \mathrm{p}<0.05,{ }^{* *} \mathrm{p}<0.01$ by one-way analysis of variance (ANOVA). 
Post hoc tests revealed that LLs in the 50-59 years and $\geq 60$ years age groups were significantly lower compared to those in the $<50$ years age group ( $\mathrm{p}=0.014$ and $\mathrm{p}<0.001$, respectively). L2-LSLs in the 50-59 years and $\geq 60$ years age groups were significantly lower compared to those in the $<50$ years age group $(\mathrm{p}=0.013$ and $\mathrm{p}<0.001$, respectively). L3-LSLs in the $\geq 60$ years age group were significantly lower compared to those in the $<50$ years age group ( $\mathrm{p}=0.012$ ). L4-LSLs in the $50-59$ years and $\geq 60$ years age groups were significantly lower compared to those in the $<50$ years age group $(\mathrm{p}=0.001$ and $\mathrm{p}=0.001$, respectively) (Table 2).

\section{LBP and lumbar sagittal alignment}

About $14 \%$ of subjects $(n=82)$ satisfied the definition of clinically meaningful LBP that lasted more than 1 week or occurred more frequently than once a month with a severe degree of pain. In the presence of LBP, LL and L4LSL were significantly lower compared to subjects without LBP. There was no significant relationship between

Table 3. LBP and lumbar sagittal alignment

\begin{tabular}{|cccc}
\hline & LBP $(\mathbf{n = 8 2})$ & No LBP $(\mathbf{n}=\mathbf{4 8 7})$ & p-value \\
\hline LL & $45.9 \pm 13.4$ & $50.4 \pm 11.4$ & $0.001^{* *}$ \\
\hline L1-LSL & $1.3 \pm 4.9$ & $2.1 \pm 4.2$ & 0.161 \\
\hline L2-LSL & $5.8 \pm 4.9$ & $5.8 \pm 3.8$ & 0.958 \\
L3-LSL & $7.8 \pm 4.9$ & $8.9 \pm 3.8$ & 0.060 \\
\hline L4-LSL & $11.3 \pm 5.4$ & $12.8 \pm 4.7$ & $0.011^{*}$ \\
\hline L5-LSL & $19.4 \pm 7.1$ & $20.6 \pm 6.4$ & 0.165 \\
\hline SS & $32.5 \pm 8.3$ & $34.3 \pm 7.9$ & 0.054 \\
\hline
\end{tabular}

Values are presented as mean \pm standard deviation. LBP, low back pain; LL, lumbar lordosis; LSL, lumbar segmental lordosis; SS, sacral slope.

${ }^{*} \mathrm{p}<0.05,{ }^{* *} \mathrm{p}<0.01$.
LBP and SS or the other LSLs (Table 3).

\section{DSN and lumbar sagittal alignment}

Lumbar DSN was most frequently observed in L5/S1 (69.6\%) and L4/5 (45.7\%). The rate of DSN was $9.7 \%$ in $\mathrm{L} 1 / 2,13.9 \%$ in $\mathrm{L} 2 / 3$, and $23.7 \%$ in $\mathrm{L} 3 / 4$ (Table 1 ). In the presence of DSN at any level from L1/2 to L5/S1, LL and SS were significantly smaller compared to subjects without DSN ( $\mathrm{p}<0.05)$ (Table 4).

There was also negative correlation between grade of DSN at any level from L1/2 to L5/S1 and LL and SS. There was negative correlation between grades of L1/2 DSN and L1-LSL (-0.205), L2-LSL (-0.168), and L5-LSL $(-0.13)$. There was negative correlation between grades of L2/3 DSN and L1-LSL (-0.153), L2-LSL (-0.267), L3-LSL $(-0.223)$, and L4-LSL (-0.13). There was negative correlation between grades of L3/4 DSN and L3-LSL (-0.292) and L4-LSL (-0.241). There was negative correlation between grades of L4/5 DSN and L4-LSL (-0.388) and L5LSL (-0.157) and positive correlation with L1-LSL (0.095). There was negative correlation between grades of L5/S1 DSN and L4-LSL (-0.084) and L5-LSL (-0.456) and positive correlation with L2-LSL (0.123) (Table 5).

The association between LSLs of L1 to L5 segments with DSN of L1/2 to L5/S1 are summarized in Fig. 2. The segmental lordotic angle was markedly reduced at the level of DSN. In the presence of L1/2 DSN, LSL was significantly decreased at L1, L2, L3, and L4 segments, with the largest change in $\mathrm{Ll}(\Delta-3.99)$. In the presence of $\mathrm{L} 2 / 3$ DSN, LSL was significantly decreased at L1, L2, L3, and L4 segments, with the largest change at L2 $(\Delta-3.31)$. In the presence of L3/4 DSN, LSL was significantly decreased at L3 and L4 with the largest change at L3 $(\Delta-2.87)$.

DSNs of the lowest lumbar levels, L4/5 and L5/S1, was associated with increased lordotic angles in the distant

Table 4. Comparison of lumbar lordosis and sacral slope in subjects with and without DSN

\begin{tabular}{lccccccc}
\hline \multirow{2}{*}{ Level of DSN } & \multicolumn{3}{c}{${\text { Lumbar lordosis }\left({ }^{\circ}\right)}$} & & \multicolumn{3}{c}{ Sacral slope $\left(^{\circ}\right)$} \\
\cline { 2 - 3 } \cline { 6 - 7 } & DSN & No DSN & p-value & & DSN & No DSN & p-value \\
\hline L1/2 & $41.4 \pm 14.9$ & $50.7 \pm 11.1$ & $<0.001$ & & $29.0 \pm 7.3$ & $34.6 \pm 7.8$ & $<0.001$ \\
L2/3 & $40.4 \pm 12.2$ & $51.3 \pm 11.0$ & $<0.001$ & & $29.7 \pm 7.2$ & $34.7 \pm 7.9$ & $<0.001$ \\
L3/4 & $44.3 \pm 12.7$ & $51.5 \pm 11.0$ & $<0.001$ & & $30.9 \pm 7.7$ & $35.0 \pm 7.8$ & $<0.001$ \\
L4/5 & $47.5 \pm 12.3$ & $51.7 \pm 11.1$ & $<0.001$ & & $32.8 \pm 7.7$ & $35.1 \pm 8.1$ & 0.001 \\
\hline L5/S1 & $48.9 \pm 11.7$ & $51.8 \pm 11.8$ & 0.008 & & $33.4 \pm 7.9$ & $35.3 \pm 7.8$ & 0.009 \\
\hline
\end{tabular}

Values are presented as mean \pm standard deviation.

DSN, disc space narrowing. 
Table 5. Spearman correlation coefficients $(\rho)$ between lumbar sagittal alignment and grades of DSN

\begin{tabular}{|c|c|c|c|c|c|c|c|c|c|c|}
\hline & \multicolumn{2}{|c|}{ L1/2 DSN } & \multicolumn{2}{|c|}{ L2/3 DSN } & \multicolumn{2}{|c|}{ L3/4 DSN } & \multicolumn{2}{|c|}{ L4/5 DSN } & \multicolumn{2}{|c|}{ L5/S1 DSN } \\
\hline & $\rho$ & p-value & $\rho$ & p-value & $\rho$ & p-value & $\rho$ & p-value & $\rho$ & p-value \\
\hline LL & -0.199 & $<0.001^{* *}$ & -0.314 & $<0.001^{* *}$ & -0.263 & $<0.001^{* *}$ & -0.231 & $<0.001^{* *}$ & -0.216 & $<0.001^{* *}$ \\
\hline L1-LSL & -0.259 & $<0.001^{* *}$ & -0.153 & $<0.001^{* *}$ & 0.031 & 0.465 & 0.095 & $0.023^{*}$ & 0.062 & 0.136 \\
\hline L2-LSL & -0.168 & $<0.001^{* *}$ & -0.267 & $<0.001^{* *}$ & -0.072 & 0.087 & 0.012 & 0.773 & 0.123 & $0.003^{* *}$ \\
\hline L3-LSL & -0.082 & 0.052 & -0.223 & $<0.001^{* *}$ & -0.292 & $<0.001^{* *}$ & -0.054 & 0.200 & 0.073 & 0.081 \\
\hline L4-LSL & -0.096 & $0.022^{*}$ & -0.130 & $0.002^{* *}$ & -0.241 & $<0.001^{* *}$ & -0.388 & $<0.001^{* *}$ & -0.084 & $0.044^{*}$ \\
\hline L5-LSL & 0.010 & 0.806 & -0.024 & 0.562 & -0.075 & 0.075 & -0.157 & $<0.001^{* *}$ & -0.456 & $<0.001^{* *}$ \\
\hline SS & -0.205 & $<0.001^{* *}$ & -0.217 & $<0.001^{* *}$ & -0.227 & $<0.001^{* *}$ & -0.203 & $<0.001^{* *}$ & -0.187 & $<0.001^{* *}$ \\
\hline
\end{tabular}

DSN, disc space narrowing; LL, lumbar lordosis; LSL, lumbar segmental lordosis; SS, sacral slope.

${ }^{*} \mathrm{p}<0.05,{ }^{* *} \mathrm{p}<0.01$.

(A)

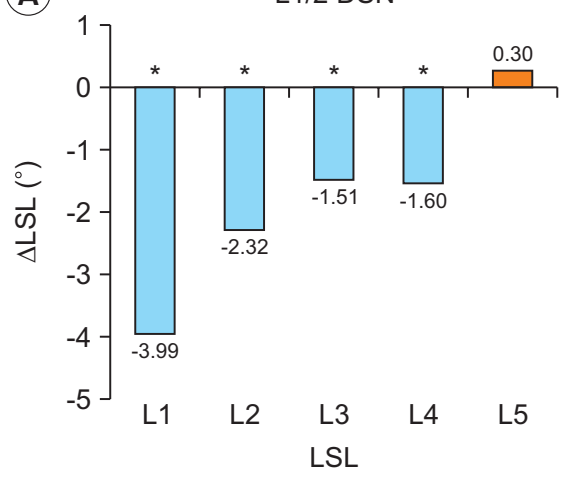

(D)

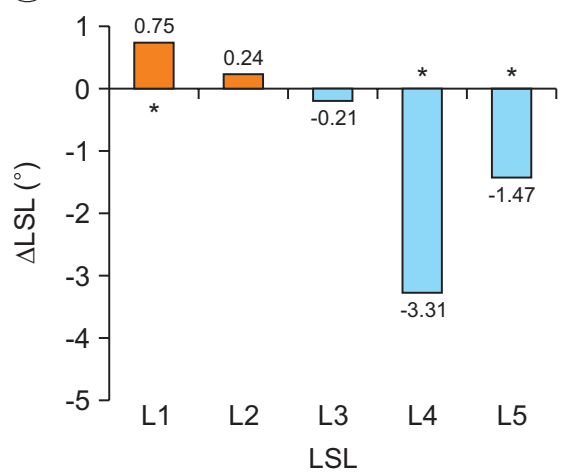

(B)

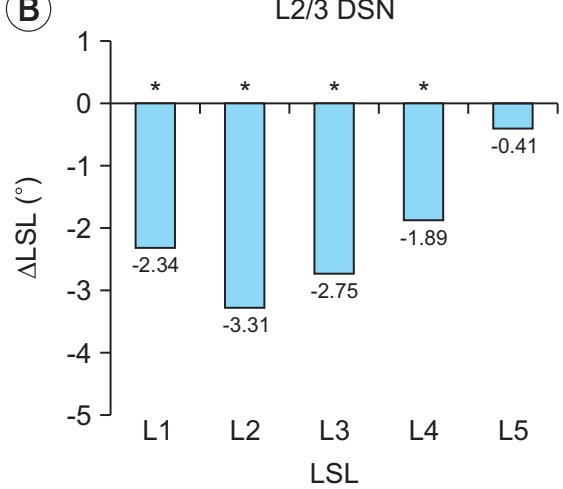

(E)

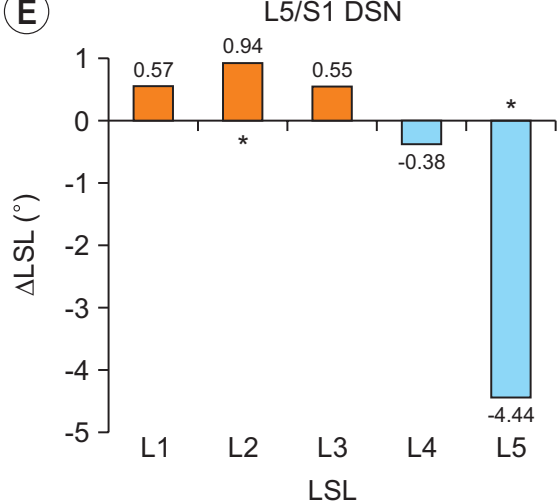

(C)

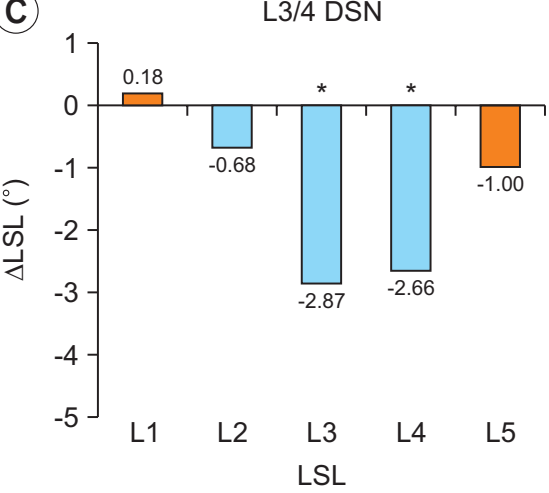

Fig. 2. Difference in LSL values ( $\triangle$ LSLs) according to the presence or absence of DSN grades: (A) L1/2 DSN, (B) L2/3 DSN, (C) L3/4 DSN, (D) L4/5 DSN, and (E) L5/S1 DSN. LSL, lumbar segmental lordosis; DSN, disc space narrowing. ${ }^{*} \mathrm{p}<0.05$ using Student t-test. $\Delta$ LSL $\left(^{\circ}\right)=$ LSL with DSN - LSL without DSN.

lumbar levels, in addition to the reduced lordotic angle at that level. In the presence of L4/5 DSN, LSL was significantly decreased at L4 and L5 segments with the largest change at L4 $(\Delta-3.31)$, while L1-LSL was increased inversely $(\Delta 0.75)$. In the presence of L5/S1 DSN, LSL was significantly decreased at L5 segment $(\Delta-4.44)$, while L2-
LSL was increased inversely $(\Delta 0.94)$.

Reversing the results, L1-LSL decrease showed significant relationship with L1/2 and L2/3 DSN. Decrease of L2-LSL was related with L1/2 and L2/3 DSN. L3-LSL was related to L1/2, L2/3, and L3/4 DSN. L4-LSL was related to $\mathrm{L} 1 / 2, \mathrm{~L} 2 / 3, \mathrm{~L} 3 / 4$, and L4/5 DSN. L5-LSL was related to 


\section{L4/5 and L5/S1 DSN.}

\section{DISCUSSION}

In this study, we compared sagittal plane alignment between sexes and different age groups, in subjects with and without LBP, and in subjects with and without DSN. In our study, sagittal plane alignment was significantly associated with all four factors: sex, age, LBP, and DSN. There was a compensational increase in LSL distant to the DSN, while LSLs around the levels of DSN were decreased to maintain the overall LL.

LL is a unique aspect of the human body that facilitates upright posture and bipedal walking [1]. When the human body evolved for upright bipedal walking, the human spine was repositioned to a new location and LL developed to facilitate efficient bipedal walking [1]. Approximately $90 \%$ of LL is delivered by wedging of the discs while the remaining $10 \%$ is due to wedging of the vertebral bodies [28]. This means that the discs play an important role in maintaining LL. Two-thirds of LL occurs at L4 and L5 segments [13]. The discs also carry the greatest load in the spine [29], and DSN is most commonly observed at L4/5 and L5/S1 level [3,14]. In this study, we found similar results that lordotic angle between L4S1 accounted for a total of $66 \%$ of LL angle and lumbar DSN occurred most frequently in L5/S1 (69.6\%) and L4/5 (45.7\%).

The relationship between sex and LL is controversial. There are two opinions. One is that there is no difference in LL between the sexes $[5,13,14]$, and the other is that LL is greater in female $[15,16]$. In our study, LL was similar between the sexes. We studied the differences in LSL as well as LL according to sex. We found that male had a greater LSL at two lower lumbar levels and female had a greater LSL at two high lumbar levels. According to the lumbar spinal curvature model derived from 158 individuals, the spine in female were more backwardly (dorsally) curved than their upper lumbar spine although the amount of lordosis was similar between sexes [30]. This difference in the morphology of the spine in female could be closely related to pregnancy. During pregnancy, as a compensatory mechanism, LL increases gradually to account for fetal load until birth [31]. The center of mass gradually moves forward and the upper part of a female's lumbar curve is positioned further backward [30,32].
Similarly, LSLs in the upper lumbar level are greater in female.

In our study, LL and LSL gradually decreased with age. The relationship between age and LL is conflicting according to the age of study population. Some studies have reported no association between age and LL $[5,13]$. Other studies have claimed that LL decreases with age $[17,18]$. The studies which concluded that there was no relationship between age and LL differed from our study regarding age distribution. One study involved relatively younger individuals aged between 20 and 65 years [13], and the other study involved relatively older individuals aged between 50 and 85 years [5]. The subjects in our study ranged from 39 to 82 years. Differences in the age distribution of study subjects between studies may result in discrepancies in the findings of the relationship between age and lordotic angle.

Decreasing lordotic angle with age could be related with multi-level DSN. Skaf et al. [14] reported that young patients had lower lumbar levels of disc herniation whereas older patients had upper lumbar levels of disc herniation with smaller lumbar lordotic angle. With aging, disc herniation starts at the lower lumbar vertebra and moves cranially $[14,33]$. DSN made reducing LSL at the same level and multi-level DSN leads to a decrease in LSLs and overall LL.

In this study, in the presence of DSN, both LL and SS were decreased. Regarding the association between DSN and LSL, the decrease in lordosis occurred mostly around the level of DSN, and LSL was increased at distant levels in the presence of L4/5 and L5/S1 DSN, suggesting compensatory lordotic changes. Similar results were also obtained through the Spearman correlation analysis between grades of DSN and LL, LSLs and SS.

The gravity line is normally located anterior to the spine to support human bipedalism. For several reasons, the gravity line moves forward [34]. These reasons include abdominal hypertrophy, pregnancy, thoracic kyphosis, and loss of LL. The human body tries to align with the gravity line through several compensatory mechanisms [35]. Loss of LL is corrected by increased pelvic tilt, knee flexion, and thoracic lordosis [35]. Takeda et al. [18] reported that the cause of loss of LL was changes in anterior wedging of the lumbar discs, which is the same as decreasing LSL. They also reported that individuals who have anterior wedging at L2-3 and L3-4 also have poste- 
rior wedging at L5-S1 that possibly compensates to maintain overall LL [18].

A recent systematic review and meta-analysis on the relationship between LBP and LL showed that patients with LBP tended to have small lumbar lordotic angles than healthy individuals [6]. In our study, LL and L4-LSL were significantly lower in subjects with LBP. L3-LSL showed a tendency to be lower in subjects with LBP, but the difference was not significant. Based on our results, L4-LSL was significantly related to DSN at four levels $(\mathrm{L} 1 / 2, \mathrm{~L} 2 / 3$, L3/4, and L4/5) and L3-LSL was related to DSN at three levels (L1/2, L2/3, and L3/4). In addition, L5-LSL was related to DSN at two levels (L4/5 and L5/S1). These results showed that loss of L4-LSL more accurately reflected extended multilevel DSN, which was also associated with LBP. Furthermore, a prospective study that enrolled a total of 403 healthcare workers aged 18-40 years who had never experienced serious LBP in their lifetime reported that loss of LL is a risk factor for LBP [36]. After 3 years, 90 participants reported serious LBP, for which, loss of LL was found to be a risk factor [36].

It has been reported that LL recovers to almost normal and LBP improves in lumbar degenerative disc disorders following surgical fusion treatment [37]. Endo et al. [8] reported that restoration of LL was correlated with postoperative symptoms. Surgical treatment was performed on 61 symptomatic lumbar disc herniation patients, and results were compared with 60 age-matched healthy participants without LBP. Before surgery, the average LL of patients was lower than that of the control group. Six months after surgery, patients showed significant recovery of LBP and an increase in LL. The degree of LL was correlated with the subjective symptoms after surgery [8].

There were several limitations to this study. First, there may have been selection bias because only farmers in Gangwon Province, South Korea were recruited. Therefore, it may be difficult to generalize our results to the general population. Second, self-reported questionnaires are associated with recall bias because subjects may not remember LBP or remember inaccurately, and this may have influenced the accuracy of the responses provided. Third, because there was no longitudinal data, we were unable to follow up the participant's change of sagittal plane alignment and LBP. Fourth, the spine radiograph was taken only from the lumbar spine and did not include the whole spine. Fifth, our study does not include one of the important spinopelvic parameters of the sagittal vertical axis. Lastly, we did not consider the presence of diseases related to the spine. The presence of thoracolumbar compression fractures can affect spinopelvic parameters such as LL and LSL. Additionally, the presence of spondylolysis and spondylolisthesis can cause LBP. It is possible that these diseases have influenced our study.

In conclusion, sagittal plane alignment was associated with sex, age, LBP, and DSN. Male had a greater LSL at lower lumbar levels and female had a greater LSL at upper lumbar levels. LL, LSL were decreased with age. LL and L4-LSL were significantly lower in subjects with LBP. Decreased LL occurred at the level of DSN, and a compensative increase in LL in the lumbar segments distant to the decreased lordosis segment. The presence of decreased LL could be a sign of a lumbar disc disorder. In the presence of DSN, LSLs were lower around the level of DSN, but LSLs at a distant level were higher to maintain the overall LL. Assessment of sagittal plane alignment can provide information about the pathophysiology of LBP and disc herniation. Clinically, lumbar disc disorder should be suspected if decrease in LL, LSL and DSN at a certain level are seen. Further studies are required to confirm the influence of sex, age, LBP, and DSN on sagittal plane alignment.

\section{CONFLICT OF INTEREST}

No potential conflict of interest relevant to this article was reported.

\section{ACKNOWLEDGMENTS}

This study was supported by Research Grant for Center for Farmers' Safety and Health at Kangwon National University Hospital from Korean Ministry of Agriculture, Food and Rural Affairs.

\section{AUTHOR CONTRIBUTION}

Conceptualization: Baek S, Park KH. Methodology: Baek S, Kang EK, Park HW, Kim G, Kim SH. Funding acquisition: Baek S. Writing - original draft: Baek S, Park KH. Writing - review and editing: Baek S, Park KH. Approval of final manuscript: all authors. 


\section{REFERENCES}

1. Filler AG. Emergence and optimization of upright posture among hominiform hominoids and the evolutionary pathophysiology of back pain. Neurosurg Focus 2007;23:E4.

2. Legaye J, Duval-Beaupere G. Sagittal plane alignment of the spine and gravity: a radiological and clinical evaluation. Acta Orthop Belg 2005;71:213-20.

3. de Schepper EI, Damen J, van Meurs JB, Ginai AZ, Popham M, Hofman A, et al. The association between lumbar disc degeneration and low back pain: the influence of age, gender, and individual radiographic features. Spine (Phila Pa 1976) 2010;35:531-6.

4. Luoma K, Riihimaki H, Luukkonen R, Raininko R, Viikari-Juntura E, Lamminen A. Low back pain in relation to lumbar disc degeneration. Spine (Phila Pa 1976) 2000;25:487-92.

5. Tsuji T, Matsuyama Y, Sato K, Hasegawa Y, Yimin Y, Iwata $\mathrm{H}$. Epidemiology of low back pain in the elderly: correlation with lumbar lordosis. J Orthop Sci 2001;6:307-11.

6. Chun SW, Lim CY, Kim K, Hwang J, Chung SG. The relationships between low back pain and lumbar lordosis: a systematic review and meta-analysis. Spine J 2017;17:1180-91.

7. Barrey C, Darnis A. Current strategies for the restoration of adequate lordosis during lumbar fusion. World J Orthop 2015;6:117-26.

8. Endo K, Suzuki H, Tanaka H, Kang Y, Yamamoto K. Sagittal spinal alignment in patients with lumbar disc herniation. Eur Spine J 2010;19:435-8.

9. Roussouly P, Gollogly S, Berthonnaud E, Dimnet J. Classification of the normal variation in the sagittal alignment of the human lumbar spine and pelvis in the standing position. Spine (Phila Pa 1976) 2005;30:346-53.

10. Abbas J, Hamoud K, May H, Hay O, Medlej B, Masharawi Y, et al. Degenerative lumbar spinal stenosis and lumbar spine configuration. Eur Spine J 2010;19:1865-73.

11. Murata Y, Takahashi K, Hanaoka E, Utsumi T, Yamagata $\mathrm{M}$, Moriya $\mathrm{H}$. Changes in scoliotic curvature and lordotic angle during the early phase of degenerative lumbar scoliosis. Spine (Phila Pa 1976) 2002;27:226873.
12. Quint U, Wilke HJ. Grading of degenerative disk disease and functional impairment: imaging versus patho-anatomical findings. Eur Spine J 2008;17:170513.

13. Jackson RP, McManus AC. Radiographic analysis of sagittal plane alignment and balance in standing volunteers and patients with low back pain matched for age, sex, and size: a prospective controlled clinical study. Spine (Phila Pa 1976) 1994;19:1611-8.

14. Skaf GS, Ayoub CM, Domloj NT, Turbay MJ, El-Zein C, Hourani MH. Effect of age and lordotic angle on the level of lumbar disc herniation. Adv Orthop 2011;2011:950576.

15. Vialle R, Levassor N, Rillardon L, Templier A, Skalli W, Guigui P. Radiographic analysis of the sagittal alignment and balance of the spine in asymptomatic subjects. J Bone Joint Surg Am 2005;87:260-7.

16. Murrie VL, Dixon AK, Hollingworth W, Wilson H, Doyle TA. Lumbar lordosis: study of patients with and without low back pain. Clin Anat 2003;16:144-7.

17. Amonoo-Kuofi HS. Changes in the lumbosacral angle, sacral inclination and the curvature of the lumbar spine during aging. Acta Anat (Basel) 1992;145:373-7.

18. Takeda N, Kobayashi T, Atsuta Y, Matsuno T, Shirado $\mathrm{O}$, Minami A. Changes in the sagittal spinal alignment of the elderly without vertebral fractures: a minimum 10-year longitudinal study. J Orthop Sci 2009;14:74853.

19. Fernand R, Fox DE. Evaluation of lumbar lordosis: a prospective and retrospective study. Spine (Phila Pa 1976) 1985;10:799-803.

20. Been E, Kalichman L. Lumbar lordosis. Spine J 2014;14:87-97.

21. Harrison DE, Harrison DD, Cailliet R, Janik TJ, Holland B. Radiographic analysis of lumbar lordosis: centroid, Cobb, TRALL, and Harrison posterior tangent methods. Spine (Phila Pa 1976) 2001;26:E235-42.

22. Cho IY, Park SY, Park JH, Kim TK, Jung TW, Lee HM. The effect of standing and different sitting positions on lumbar lordosis: radiographic study of 30 healthy volunteers. Asian Spine J 2015;9:762-9.

23. Lord MJ, Small JM, Dinsay JM, Watkins RG. Lumbar lordosis: effects of sitting and standing. Spine (Phila Pa 1976) 1997;22:2571-4.

24. Dunn G. Design and analysis of reliability studies. Stat Methods Med Res 1992;1:123-57. 
25. Mimura M, Panjabi MM, Oxland TR, Crisco JJ, Yamamoto I, Vasavada A. Disc degeneration affects the multidirectional flexibility of the lumbar spine. Spine (Phila Pa 1976) 1994;19:1371-80.

26. Kim D. Effect of musculoskeletal pain of care workers on job satisfaction. J Phys Ther Sci 2018;30:164-8.

27. Baron S, Hales T, Hurrell J. Evaluation of symptom surveys for occupational musculoskeletal disorders. Am J Ind Med 1996;29:609-17.

28. Been E, Barash A, Marom A, Kramer PA. Vertebral bodies or discs: which contributes more to human-like lumbar lordosis? Clin Orthop Relat Res 2010;468:1822-9.

29. Ghezelbash F, Shirazi-Adl A, Arjmand N, El-Ouaaid Z, Plamondon A, Meakin JR. Effects of sex, age, body height and body weight on spinal loads: sensitivity analyses in a subject-specific trunk musculoskeletal model. J Biomech 2016;49:3492-501.

30. Hay O, Dar G, Abbas J, Stein D, May H, Masharawi Y, et al. The lumbar lordosis in males and females, revisited. PLoS One 2015;10:e133685.

31. Whitcome KK, Shapiro LJ, Lieberman DE. Fetal load and the evolution of lumbar lordosis in bipedal homi- nins. Nature 2007;450:1075-8.

32. Masharawi Y, Dar G, Peleg S, Steinberg N, Medlej B, May $\mathrm{H}$, et al. A morphological adaptation of the thoracic and lumbar vertebrae to lumbar hyperlordosis in young and adult females. Eur Spine J 2010;19:76873.

33. Dammers R, Koehler PJ. Lumbar disc herniation: level increases with age. Surg Neurol 2002;58:209-13.

34. Schwab F, Lafage V, Boyce R, Skalli W, Farcy JP. Gravity line analysis in adult volunteers: age-related correlation with spinal parameters, pelvic parameters, and foot position. Spine (Phila Pa 1976) 2006;31:E959-67.

35. Lamartina C, Berjano P. Classification of sagittal imbalance based on spinal alignment and compensatory mechanisms. Eur Spine J 2014;23:1177-89.

36. Adams MA, Mannion AF, Dolan P. Personal risk factors for first-time low back pain. Spine (Phila Pa 1976) 1999;24:2497-505.

37. Pavlov PW, Meijers H, van Limbeek J, Jacobs WC, Lemmens JA, Obradov-Rajic M, et al. Good outcome and restoration of lordosis after anterior lumbar interbody fusion with additional posterior fixation. Spine (Phila Pa 1976) 2004;29:1893-1900. 